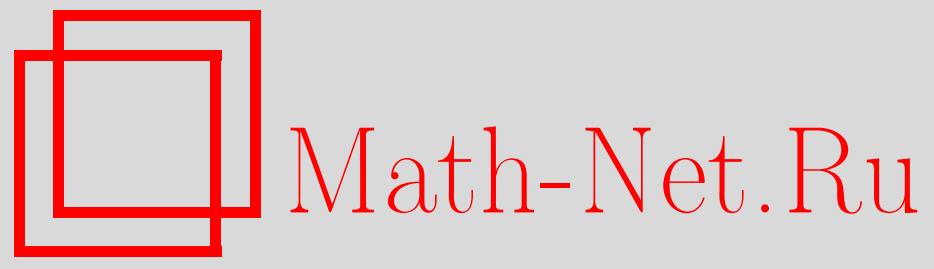

А. С. Мищенко, Дискретный процесс Бесселя и его свойства, Теория вероятн. и ее примен., 2005, том 50, выпуск 4, 797-806

DOI: https://doi.org/10.4213/tvp136

Использование Общероссийского математического портала Math-Net.Ru подразумевает, что вы прочитали и согласны с пользовательским соглашением

http: //www . mathnet.ru/rus/agreement

Параметры загрузки:

IP: 54.197 .130 .99

26 апреля 2023 г., 11:46:50

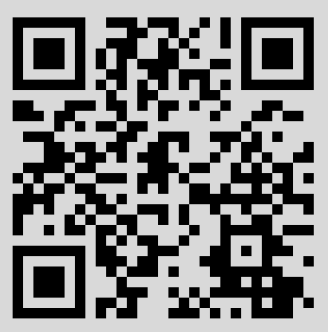




\section{ДИСКРЕТНЫЙ ПРОЦЕСС БЕССЕЛЯ И ЕГО СВОЙСТВА}

В данной работе рассматривается дискретный аналог процесса Бесселя размерности три - некоторый дискретный процесс, сходящийся к непрерывному процессу Бесселя в смысле принципа инвариантности Цонскера-Прохорова и имеющий столь же элементарную структуру траектории, как и у простейшего случайного блуждания.

В работе дается четыре эквивалентных определения дискретного процесса Бесселя, которые освещают его с различных точек зрения. Изучение этого процесса показывает, что взаимосвязи между ним и простейшим случайным блужданием полностью повторяют известные соотношения, связывающие процесс Бесселя и броуновское движение в непрерывном времени. Так, в работе формулируются и доказываются дискретные варианты теоремы Питмана, разложения Вильямса и других известных свойств указанных процессов.

Ключевые слова и фразы: процесс Бесселя, случайное блуждание, дискретные аналоги, теорема Питмана, теорема Леви, разложение Вильямса.

1. Введение. Пусть $S_{n}$ обозначает простейшее симметричное случайное блуждание, т.е. $S_{n}:=\sum_{i=1}^{n} \xi_{i}$, где $\left\{\xi_{i}\right\}_{i=1,2, \ldots}$ - независимые случайные величины, имеющие бернуллиевское симметричное распределение $\mathbf{P}\left\{\xi_{i}= \pm 1\right\}=\frac{1}{2}$.

$\mathrm{K}$ процессу $\left(S_{n}\right)$ применима хорошо известная теорема, которая носит название принципа инвариантности Донскера-Прохорова [2, гл. ХІІІ, теорема 1.9]. Суть этого результата состоит в следующем.

Предложение 1 (принцип инвариантности Донскера-Прохорова). Рассмотрим последовательность независимых одинаково распределенных случайных величин $\left(\eta_{n}\right)_{n=1,2, \ldots}, \mathbf{E} \eta_{n}=0, \mathbf{E} \eta_{n}^{2}=1$ и случайное блуждание $S_{n}:=\sum_{k=1}^{n} \eta_{k}$. По $S_{n}$ построим случайный прочесс в непрерывном времени с помощью кусочно-линейной интерполячии, который тажже обозначим через $\left(S_{t}\right)_{t \geqslant 0}$. Тогда $\left(S_{m t} / \sqrt{m}\right)_{t \geqslant 0} \stackrel{\mathrm{w}}{\longrightarrow}$ $\left(B_{t}\right)_{t \geqslant 0}$ при $m \rightarrow \infty$ в смысле слабой сходимости распределений в пространстве $\mathscr{C}[0, \infty)$, где $\left(B_{t}\right)_{t \geqslant 0}-$ стандартное броуновское движение.

Таким образом, с одной стороны, процесс $\left(S_{n}\right)$ имеет крайне простую структуру траекторий, к нему применимы алгебраические, комбинаторные методы рассуждения (биекции на множестве траекторий и тому подобное). С другой стороны, с помощью принципа инвариантности результаты, полученные для случайного блуждания, могут быть перенесены на случай непрерывного времени. Вспомним, например, насколько просто доказывается в дискретном случае принцип отражения, а затем этот результат можно легко перенести на случай броуновского движения благодаря теореме Донскера-Прохорова.

Помимо броуновского движения теория вероятностей изучает множество других связанных с ним процессов. В этой статье для нас особый интерес представляет процесс Бесселя размерности три, который мы будем обозначать $\left(R_{t}\right)_{t \geqslant 0}$. Этот процесс подробно изучается во многих работах, в книге [2] ему посвящен $\S 3$ главы VI. Одно из эквивалентных его определений таково: процессом Бесселя размерности три называется модуль трехмерного броуновского движения.

* Московский государственный университет им. М.В. Ломоносова, механикоматематический факультет, кафедра теории вероятностей, Ленинские горы, 119992 Москва, Россия; e-mail: andrei.mischenko@mail.ru 
В знаменитой работе Дж. Питмана [1] рассматривался некоторый дискретный процесс, который вправе считаться дискретным аналогом процессом Бесселя размерности три. Он имеет столь же простую структуру траекторий, как и простейшее случайное блуждание, и, согласно результатам этой работы, сходится к процессу $\left(R_{t}\right)_{t \geqslant 0}$ в смысле принципа инвариантности. Мы будем обозначать этот процесс через $\left(R_{n}\right)_{n=0,1, \ldots}$.

Помимо определения, используемого Питманом, в п. 2 мы приводим три других определения и доказываем их эквивалентность. Используемые при этом вспомогательные утверждения представляют интерес и сами по себе, в частности, мы формулируем и доказываем дискретный аналог хорошо известной теоремы Леви.

Последний пункт демонстрирует глубину аналогии между непрерывным и дискретным процессами Бесселя на примере дискретного варианта разложения Вильямса. Также в работе приводятся дискретные аналоги и других известных свойств непрерывного процесса Бесселя.

В действительности, теория случайных процессов рассматривает целое семейство процессов Бесселя различных размерностей, задаваемое стохастическим дифференциальным уравнением, в которое размерность входит в качестве параметра. Но мы будем работать лишь с процессом размерности три, поэтому указание на нее будет, как правило, опускаться, мы будем называть процесс $\left(R_{t}\right)$ просто процессом Бесселя, а процесс $\left(R_{n}\right)_{n=0,1, \ldots}$ дискретным процессом Бесселя.

2. Построение дискретного процесса Бесселя. В этом пункте мы дадим четыре определения дискретного процесса Бесселя $\left(R_{n}\right)_{n=0,1, \ldots}$, докажем их эквивалентность и убедимся, что для этого процесса имеет место сходимость к непрерывному процессу Бесселя $\left(R_{t}\right)_{t \geqslant 0}$ в том же смысле, что и в теореме Донскера-Прохорова.

Для простейшего случайного блуждания $\left(S_{n}\right)$ будем обозначать через $M_{n}:=$ $\sup _{k \leqslant n} S_{k}$ процесс его максимума.

О п р е д е л е н и е 1 . Зададим процесс $\left(R_{n}^{(1)}\right)_{n=0,1, \ldots}$, положив $R_{n}^{(1)}:=2 M_{n}-S_{n}$.

Определение 1 повторяет приводимое в работе [1], где доказывается следующее соотношение между броуновским движением и процессом Бесселя размерности три (см. также [2, гл. VI, теорема 3.5 , следствие 3.6]).

Предложение 2 (теорема Питмана). Пусть $\left(B_{t}\right)_{t \geqslant 0}-$ стандартное броуновское движение, а $M_{t}:=\sup _{s \leqslant t} B_{s}-$ прочесс его максимума. Пусть $\left(R_{t}\right)_{t \geqslant 0}-$ трехмерный прочесс Бесселя, a $J_{t}:=\inf _{s \geqslant t} R_{s}$. Тогда следующие пары прочессов совпадают по распределению:

$$
\left(2 M_{t}-B_{t}, M_{t}\right)_{t \geqslant 0} \stackrel{\text { law }}{=}\left(R_{t}, J_{t}\right)_{t \geqslant 0} .
$$

При этом условные распределения $\operatorname{Law}\left(J_{t} \mid \mathscr{F}_{t}\right)$ u Law $\left(R_{t}-J_{t} \mid \mathscr{F}_{t}\right)$ являются равномерными распределениями на отрезке $\left[0, R_{t}\right]$, где $\mathscr{F}_{t}:=\sigma\left(R_{s}, s \leqslant t\right)$.

В свете этого результата кажется весьма естественным назвать процесс $R_{n}^{(1)}$ дискретным процессом Бесселя. $\mathrm{K}$ тому же из свойства возвратности случайного блуждания мгновенно следует соотношение $M_{n}=\inf _{k \geqslant n} R_{k}^{(1)}$. Свойство равномерности условного распределения также имеет место в дискретном случае, что будет доказано чуть позже.

А сейчас рассмотрим процесс $L_{n}:=\sum_{i=1}^{n} \mathbb{I}_{\left\{\left(S_{n-1}-1 / 2\right)\left(S_{n}-1 / 2\right)<0\right\}}$, который «считает» количество пересечений уровня $\frac{1}{2}$ случайным блужданием $\left(S_{n}\right)$. Можно считать, что для случайного блуждания этот процесс играет ту же роль, что и локальное время в нуле для броуновского движения, и вот почему. В теории броуновского движения хорошо известна теорема Леви [2, гл. IV, теорема 2.3].

Предложение 3 (теорема Леви). Для стандартного броуновского движения $\left(B_{t}\right)_{t \geqslant 0}$, прочесса его максимума $\left(M_{t}\right)_{t \geqslant 0}$ и его локального времени в нуле $\left(L_{t}\right)_{t \geqslant 0}$ имеет место следующее раєенство по распределению:

$$
\left(M_{t}-B_{t}, M_{t}\right)_{t \geqslant 0} \stackrel{\text { law }}{=}\left(\left|B_{t}\right|, L_{t}\right)_{t \geqslant 0} .
$$

Оказывается аналогичное равенство можно доказать и в дискретном случае. 
Лемма 1 (дискретная теорема Леви). Для прочессов $\left(S_{n}\right)_{n=0,1, \ldots},\left(M_{n}\right)_{n=0,1, \ldots}$, $\left(L_{n}\right)_{n=0,1, \ldots}$, указанных выше, распределения следуюших дв умерных прочессов совпадают:

$$
\left(M_{n}-S_{n}, M_{n}\right)_{n=0,1, \ldots} \stackrel{\text { law }}{=}\left(\left|S_{n}-\frac{1}{2}\right|-\frac{1}{2}, L_{n}\right)_{n=0,1, \ldots} .
$$

Д о к а з а т е л ь с т в о. Заметим, что в обеих частях равенства (1) фигурируют двумерные марковские процессы, выходящие из нуля. Поэтому для доказательства леммы достаточно проверить равенство переходных функций.

Итак, пусть $\Delta_{n}^{1}:=\left(M_{n+1}-S_{n+1}\right)-\left(M_{n}-S_{n}\right)$, а $\Delta_{n}^{2}:=M_{n+1}-M_{n}$. Исходя непосредственно из определения процессов $\left(S_{n}\right)$ и $\left(M_{n}\right)$, легко описать условное распределение этих «будущих» приращений, когда значение самого двумерного процесса $\left(M_{n}-S_{n}, M_{n}\right)$ в «настоящий» момент времени $n$ считается известным.

Если $M_{n}-S_{n}=0$, то случайное блуждание находится в точке своего максимума и на следуюшем шаге либо с вероятностью $\frac{1}{2}$ значение максимума увеличится на единицу, и тогда $\left(\Delta_{n}^{1}, \Delta_{n}^{2}\right)=(0,1)$, либо же случайное блуждание сделает шаг вниз, при этом $\left(\Delta_{n}^{1}, \Delta_{n}^{2}\right)=(1,0)$.

Напротив, если $M_{n}-S_{n}>0$, то в такой ситуации максимум не может увеличиться на следующем шаге, и, значит, $\Delta_{n}^{2}=0$ с вероятностью 1 . При этом $\Delta_{n}^{1}$ принимает значения \pm 1 с вероятностями $\frac{1}{2}$.

Аналогично, пусть $\Delta_{n}^{3}:=\left|S_{n+1}-\frac{1}{2}\right|-\left|S_{n}-\frac{1}{2}\right|$ и $\Delta_{n}^{4}:=L_{n+1}-L_{n}$.

Рассмотрим случай, когда $\left|S_{n}-\frac{1}{2}\right|=\frac{1}{2}$. Из этого равенства можно сделать вывод, что либо $S_{n}=0$, либо $S_{n}=1$. Какое именно из этих гвух значений имеет место, можно определить по четности $n$. Но в любом случае случайное блуждание пересечет на следуюшем шаге уровень $\frac{1}{2}$ с вероятностью $\frac{1}{2}$. Если это произойдет, то $L_{n}$ увеличится на 1 , а значение величины $\left|S_{n}-\frac{1}{2}\right|$ не изменится, т.е. $\left(\Delta_{n}^{3}, \Delta_{n}^{4}\right)=(0,1)$. В противном случае имеем $\left(\Delta_{n}^{1}, \Delta_{n}^{2}\right)=(1,0)$.

Пусть теперь $\left|S_{n}-\frac{1}{2}\right|>\frac{1}{2}$. Такое неравенство означает, что процесс $\left(S_{n}\right)$ находится слишком далеко от значения $\frac{1}{2}$ и, таким образом, $\Delta_{n}^{4}=0$ почти наверное, а $\Delta_{n}^{3}= \pm 1$ с равными вероятностями.

Совпадение переходных функций установлено, и, следовательно, равенство (1) действительно имеет место.

О п р е д е л е н и е 2 . Процесс $\left(R_{n}^{(2)}\right)_{n=0,1, \ldots}$ определим по следуюшей формуле: $R_{n}^{(2)}:=\left|S_{n}-\frac{1}{2}\right|-\frac{1}{2}+L_{n}$.

В дальнейшем нам понадобятся следующие обозначения. При фиксированном натуральном $N$ мы будем обозначать через $\Omega_{N}$ (конечное) множество, состоящее из всевозможных траекторий простейшего случайного блуждания $\left(S_{n}\right)_{n \leqslant N}$ до момента времени $N$. Иначе говоря, $\Omega_{N}=\left\{\omega=\left(\omega_{0}, \ldots, \omega_{N}\right)\right\}$, где $\omega_{0}=0$, а для всякого $n<N$ выполнено $\omega_{n+1}-\omega_{n}= \pm 1$. Обозначим также через $\Omega_{\infty}$ множество траекторий случайного блуждания на неограниченном интервале времени. Через $\Omega_{N}^{+} \subset \Omega_{N}$ будем обозначать подміюжество тех траекторий, которые не принимают отрицательных значений, т.е. для $\omega=\left(\omega_{0}, \ldots, \omega_{N}\right) \in \Omega_{N}^{+}$при каждом $n \leqslant N$ имеет место неравенство $\omega_{n} \geqslant 0$.

Для случайного блуждания $\left(S_{n}\right)$ рассмотрим следующие моменты времени:

$$
\begin{array}{lll}
\tau_{0}^{-}=0, & \sigma_{0}^{-}=\min \left\{k>\tau_{0}^{-} \mid S_{k}=1\right\}-1, \\
\tau_{1}^{+}=\sigma_{0}^{-}, & \sigma_{1}^{+}=\min \left\{k>\tau_{1}^{+} \mid S_{k}=0\right\}-1, \\
\tau_{1}^{-}=\sigma_{1}^{+}, & \sigma_{1}^{-}=\min \left\{k>\tau_{1}^{-} \mid S_{k}=1\right\}-1, \\
\tau_{2}^{+}=\sigma_{1}^{-}, & \sigma_{2}^{+}=\min \left\{k>\tau_{2}^{+} \mid S_{k}=0\right\}-1, \ldots .
\end{array}
$$

Точки, задаваемые формулами (2), разбивают временную ось на отрезки, которые мы будем называть положительными и отрицательными экскурсиями случайного блуждания. Это построение можно применять как для бесконечного временного интервала, так и при ограничении $\left(S_{n}\right)_{n \leqslant N}$. Во втором случае для последней экскурсии следует положить $\sigma_{k}^{ \pm}=N$. Приращением экскурсии мы будем называть разность значений на ее концах. 
Лемма 2. Зафиксируем неотрииательное число N. Можно считать, что формула из определения 2 задает некоторое отображение $\psi_{N}: \Omega_{N} \rightarrow \Omega_{N}^{+}$, которое переводит траекторию $\left(S_{n}\right)_{n \leqslant N}$ в траекторию $\left(R_{n}^{(2)}\right)_{n \leqslant N}$. Это отображение обладает следуюшим свойством. Для каждой траектории $\omega^{+} \in \Omega_{N}^{+}$, имеющей в конечный момент времени значение $x:=R_{N}^{(2)}\left(\omega^{+}\right)=\omega_{N}^{+}$, и для всякого челого у из отрезка $[0, x]$ существует и единственна траектория $\psi_{N}^{-1}\left(\omega^{+}, y\right)=\omega \in \Omega_{N}$, для которой $L_{N}(\omega)=y$, a $\psi_{N}(\omega)=\omega^{+}$.

Д о к а з а т е л ь с т о. Вообще говоря, утверждение о том, что отображение $\psi_{N}$ принимает значения в пространстве $\Omega_{N}^{+}$, нуждается в проверке. Соотношения $R_{0}^{(2)}=0$ и $R_{n}^{(2)} \geqslant 0$ очевидны, но то, что прирашения процесса $\left(R_{n}^{(2)}\right)$ равны \pm 1, сразу не видно. Однако мы не будем сейчас на этом останавливаться, вместо этого мы опишем отображение $\psi_{N}$ принципиально другим эквивалентным способом, из которого будет следовать не только это свойство, но и полное утверждение леммы.

Рассмотрим произвольную траекторию $\omega \in \Omega_{N}$ и разобьем ее на экскурсии согласно формулам (2). Далее «разрежем» траекторию на экскурсии, применим к каждой отрицательной экскурсии горизонтальную симметрию и «склеим» получившиеся отрезки траектории обратно, подвинув некоторые из них вверх, чтобы конец каждого кусочка совпадал с началом следуюшего за ним. Построенную таким образом траекторию обозначим $\widetilde{\psi}_{N}(\omega)$. Пример этого преобразования приведен на рис. $1,2$. На рис. 1 изображена некоторая траектория $\omega$, а на рис. 2 можно увидеть траекторию $\widetilde{\psi}_{N}(\omega)$, результат применения описанного отображения.

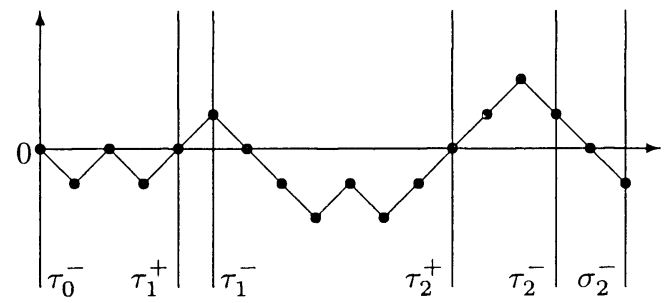

Рис. 1

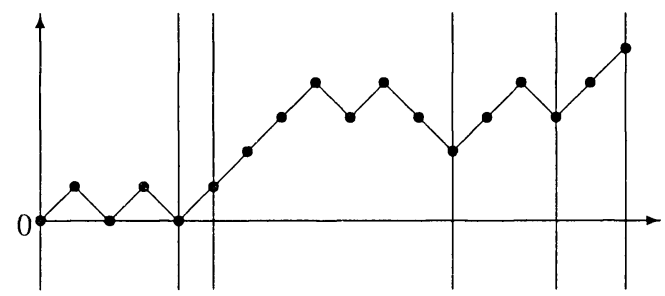

Рис. 2

Внимательно изучив формулы (2), определение процесса $\left(L_{n}\right)_{n=0,1, \ldots}$, а также определение 2 , можно усмотреть, что отображения $\psi_{N}$ и $\widetilde{\psi}_{N}$ полностью идентичны. Отсюда, в частности, видно, что отображение $\psi_{N}$ принимает значения именно в пространстве $\Omega_{N}^{+}$.

Докажем теперь существование прообраза $\psi_{N}^{-1}\left(\omega^{+}, y\right)$. Рассмотрим произвольную траекторию $\omega^{+} \in \Omega_{N}^{+}$, далее зафиксируем $y$ и найдем моменты последнего достижения траекторией $\omega^{+}$уровней $0,1, \ldots, y-1$. Другими словами, положим

$$
u_{z}=\max \left\{k \mid \omega_{k}^{+}=z-1\right\}, \quad z=1, \ldots, y,
$$

также будет удобно считать, что $u_{0}=0$, а $u_{y+1}=N$. 
Теперь «разобьем» траекторию $\omega^{+}$на отрезки $\left[u_{0}, u_{1}\right], \ldots,\left[u_{y}, u_{y+1}\right]$ и применим горизонтальную симметрию к тем из них, которые имеют нечетные номера. Если из получившихся кусочков составить траекторию $\omega$, «приставив» их по порядку друг к другу, то эти отрезки будут для такой траектории положительными и отрицательными экскурсиями. Эта траектория и есть искомый прообраз $\psi_{N}^{-1}\left(\omega^{+}, y\right)$.

Продемонстрируем это построение примером, изображенным на рис. 3,4 . В качестве $\omega^{+}$здесь была выбрана траектория из предыдущего примера (рис. 2). Как легко понять, на рис. 1 изображен ее прообраз для $y=4$. На рис. 4 с помощью описанного алгоритма построен еще один прообраз той же самой траектории, но для другого значения $y=3$.

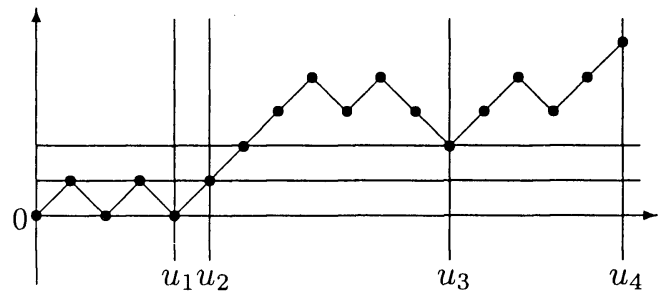

Рис. 3

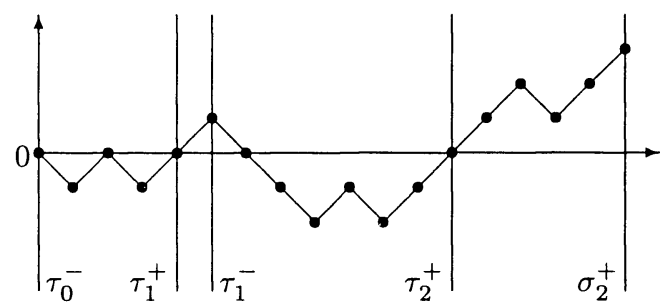

Рис. 4

Нам осталось проверить единственность такого прообраза. В принципе, она следует из того, что описанные выше построения являются в некотором смысле необходимыми. Действительно, из равенства $L_{N}(\omega)=y$ однозначно определяется знак последней экскурсии (она должна быть отрицательной для четных $y$ и положительной для нечетных), а также величина ее прирашения (его модуль должен равняться $(x-y+1)$ для $y>0$, и $x$ в случае $y=0)$. Поэтому начало этой экскурсии, т.е. точка $u_{y}$, определяется однозначно. Если взять для $u_{y}$ какое-то другое значение, отличное от формулы (3), то либо эта экскурсия будет иметь неподходящее приращение, либо отрезок $\left[u_{y}, N\right]$ вообще не будет являться экскурсией в смысле формул (2). Аналогично можно убедиться, что точки $u_{y-1}, u_{y-2}, \ldots$ тоже не могут иметь никаких других значений.

Другим, возможно более изящным, способом установить единственность прообраза $\psi_{N}^{-1}\left(\omega^{+}, y\right)$ было бы следуюшее. Сначала, воспользовавшись принципом отражения, нужно для фиксированного значения $x$ вычислить в явном виде количество траекторий $\omega^{+}$, имеющих $\omega_{N}^{+}=x$. Затем следует умножить эту величину на $(x+1)$ и просуммировать по всем возможным значениям $x$. Убедившись, что эта сумма равна $2^{N}$, можно будет сделать вывод, что множество $\Omega_{N}$ полностью исчерпывается траекториями, построенными по описанному алгоритму, для различных значений $\omega^{+}$ и $y$. Реализацию этого плана мы оставляем читателю.

А теперь рассмотрим дискретный процесс Бесселя с точки зрения теории марковских процессов. 
О п р е д е л е н и е 3 . Через $\left(R_{n}^{(3)}\right)_{n=0,1, \ldots}$ мы обозначим марковский процесс, выходящий из нуля и имеющий следуюшую переходную функцию:

$$
\mathbf{P}\left(R_{n+1}^{(3)}-R_{n}^{(3)}=\Delta \mid R_{n}^{(3)}\right)=\frac{1}{2} \frac{R_{n}^{(3)}+1+\Delta}{R_{n}^{(3)}+1}, \quad \Delta= \pm 1 .
$$

Для того чтобы дать последнее, четвертое определение дискретного процесса Бесселя, нам придется ограничиться конечным временным интервалом. Рассмотрим на множестве $\Omega_{N}^{+}$вероятностную меру $Q_{N}$, заданную формулой $Q_{N}(\{\omega\})=c_{N}\left(\omega_{N}+1\right)$. Другими словами, траектория, имеющая в последний момент времени значение $x$, наделяется весом, пропорциональным величине $(x+1)$. На основании леммы 2 можно проверить, что $c_{N}=1 / 2^{N}$.

О п р е д е л е н и е 4 . Введем $\left(R_{n}^{(4)}\right)_{n \leqslant N}$ как канонический процесс на пространстве $\Omega_{N}^{+}$, рассматриваемый относительно меры $Q_{N}$. Под каноническим понимается процесс, заданный равенством $R_{n}^{(4)}(\omega)=\omega_{n}$.

Теорема 1. Определения 1-4 эквивалентны, т.е. все четыре прочесса $\left\{\left(R_{n}^{(i)}\right)_{n=0,1, \ldots}\right\}_{i=1, \ldots, 4}$ имеют одно и то же распределение вероятностей, в дальнейшем все эти прочессы мы будет называть дискретным прочессом Бесселя и обозначать просто $\left(R_{n}\right)_{n=0,1, \ldots}$. При этом распределение прочесса $\left(R_{n}\right)$ сходится $\kappa$ распределению непрерыбного прочесса Бесселя $\left(R_{t}\right)_{t \geqslant 0}$ в смьсле принципа инвариантности Донскера-Прохорова. А именно, если обозначить через $\left(\widetilde{R}_{t}\right)_{t \geqslant 0}$ кусочно-линейную интерполячию прочесса $\left(R_{n}\right)_{n=0,1, \ldots}$, mo $\left(\widetilde{R}_{m t} / \sqrt{m}\right)_{t \geqslant 0} \stackrel{\mathrm{w}}{\longrightarrow}\left(R_{t}\right)_{t \geqslant 0}$ nри $m \rightarrow \infty$ в смысле слабой сходимости распределений в пространстве $\mathscr{C}[0, \infty)$.

Д о к а з а т е л ь с т в о. Практически все утверждения теоремы были уже доказаны выше, так что нам потребуется лишь подвести итоги. Эквивалентность определений 1 и 2 мгновенно следует из леммы 1.

Переходная функция (4) из определения 3 приводилась в работе [1], где ее вычисление базировалось на определении 1 . Мы приведем другое доказательство, отталкиваясь от определения 2. Итак проверим, что процесс $\left(R_{n}^{(2)}\right)_{n=0,1, \ldots}$ марковский и его переходная функция задается формулой (4). Зафиксируем «настоящий» момент времени $n$. Из леммы 2 видно, что при известном значении $R_{n}^{(2)}=x$ величина $L_{n}$ распределена дискретно-равномерно на отрезке $[0, x]$, причем независимо от вида траектории $\left(R_{k}^{(2)}\right)_{k \leqslant n}$. При этом, если $L_{n}=x$, то $\left|S_{n}-\frac{1}{2}\right|-\frac{1}{2}=0$ и значение на «следующем» шаге определено однозначно, $R_{n+1}^{(2)}=x+1$, поскольку $L_{n}$ не убывает, а $\left|S_{n}-\frac{1}{2}\right|-\frac{1}{2}$ неотрицательно. Вероятность такой ситуации равна $1 /(x+1)$. Если же $L_{n}<x$, что имеет место с вероятностью $x /(x+1)$, то $\left|S_{n}-\frac{1}{2}\right|-\frac{1}{2}>0$, т.е. $S_{n} \notin\{0,1\}$, следовательно, $L_{n}$ не может увеличиться на следуюшем шаге, поэтому «будушее» значение имеет два равновероятных варианта $R_{n+1}^{(2)}=x \pm 1$. Во-первых, из этих рассуждений следует марковость процесса $\left(R_{n}^{(2)}\right)$, а во-вторых, по формуле полной вероятности мы получаем переходную функцию

$$
\mathbf{P}\left(R_{n+1}^{(2)}=x+1 \mid R_{n}^{(2)}=x\right)=\frac{1}{2} \frac{x}{x+1}+\frac{1}{x+1} .
$$

Таким образом, эквивалентносาь определений 2 и 3 доказана.

Эквивалентность определений 2 и 4 напрямую следует из леммы 2. Зафиксируем «последний» момент времени $N$. При $R_{N}^{(2)}=x$ имеется ровно $(x+1)$ возможных значений для $y=L_{n}$. Все траектории пространства $\Omega_{N}$ равновероятны относительно меры $\operatorname{Law}\left(\left(S_{n}\right)_{n \leqslant N}\right)$, где $\left(S_{n}\right)$ - случайное блуждание, фигурируюшее в определении 2. Следовательно, распределение $\operatorname{Law}\left(\left(R_{n}^{(2)}\right)_{n \leqslant N}\right)$, которое индуцировано отображением $\psi_{N}$, совпадает с мерой $Q_{N}$, введенной в определении 4. Заметим, что это заодно доказывает согласованность семейства мер $\left(Q_{N}\right)_{N=0,1, \ldots}$.

Последнее утверждение теоремы, в котором говорится о сходимости к непрерывному процессу Бесселя, доказано в работе [1]. Также оно сразу следует из определения 1 , принципа инвариантности Донскера-Прохорова для броуновского движения 
(предложение 1) и теоремы Питмана (предложение 2). При этом следует воспользоваться тем, что взятие максимума является непрерывной операцией в пространстве $\mathscr{C}[0, \infty)$.

Прямым следствием полученных результатов являются следующие утверждения.

Следствие (дискретная теорема Питмана). Pассмотрим проиессь $\left(S_{n}\right)_{n=0,1, \ldots}$, $\left(M_{n}\right)_{n=0,1, \ldots}$ u $\left(R_{n}\right)_{n=0,1, \ldots}$, определенные выше. Положим $J_{n}:=\inf _{k \geqslant n} R_{k}$. Тогда следующие двумернье прочессы совпадают по распределению:

$$
\left(2 M_{n}-S_{n}, M_{n}\right)_{n=0,1, \ldots} \stackrel{\text { law }}{=}\left(R_{n}, J_{n}\right)_{n=0,1, \ldots},
$$

причем условное распределение Law $\left(J_{n} \mid F_{n}\right)$ является дискретным равномерным распределением, т.е. $J_{n}$ принимает значения $0,1, \ldots, R_{n}$ с равными вероятностями. Здесь $F_{n}:=\sigma\left(R_{k}, k=0,1, \ldots, n\right)$.

Д о к а з а те ль с т в о. Равенство распределений первых компонент указанных двумерных процессов следует из определения 1, а соотношение $M_{n}=$ $\inf _{k \geqslant n}\left(2 M_{k}-S_{k}\right)$, как уже упоминалось ранее, следует из возвратности случайного блуждания. Равномерность рассматриваемого условного распределения следует из леммы 2.

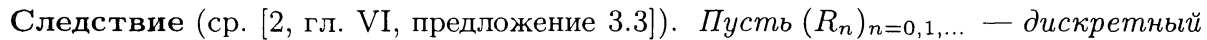
прочесс Бесселя. Тогда для любых моментов остановки $n_{1} \leqslant n_{2}$, между которыми $R_{n}$ не обращается в нуль, прочесс $\left(\left(R_{n}+1\right)^{-1}\right)_{n=n_{1}, \ldots, n_{2}}$ является мартингалом.

Доказательство состоит в непосредственной проверке мартингального свойства на основании формулы (4) определекия 3.

3. Свойства дискретного процесса Бесселя. Основная цель этого пункта сформулировать и доказать дискретный аналог теоремы Вильямса и разложения Вильямса, двух известных соотношений между броуновским движением и процессом Бесселя размерности три, точные формулировки которых можно найти, например, в [2, гл. VI, теорема 3.11 и гл. VII, теорема 4.9]. Эти результаты восходят к работе [3].

Вначале докажем несколько вспомогательных утверждений. Все они являются дискретными вариантами известных взаимосвязей между процессом Бесселя и броуновским движением. Введем необходимые обозначения. Для дискретного случайного процесса $\left(X_{n}\right)_{n=0,1, \ldots}$, принимающего значения в пространстве $\Omega_{\infty}$, и целого числа $a$ определим следующие случайные моменты времени:

$$
\begin{aligned}
l_{a}(X) & :=\inf \left\{n \mid X_{n}=a\right\}, & l_{a+}(X) & :=\inf \left\{n \mid X_{n}=a+1\right\}-1, \\
r_{a-}(X) & :=\sup \left\{n \mid X_{n}=a-1\right\}+1, & r_{a}(X) & :=\sup \left\{n \mid X_{n}=a\right\} .
\end{aligned}
$$

О п р ед е л е н и е 5 . Пусть $\left(R_{n}\right)$ - дискретный процесс Бесселя. Процесс, задаваемый равенством $R_{n}^{+}:=R_{n+r_{0}(R)}$, мы будем называть положительньлм $\partial u c-$ кретньм прочессом Бесселя.

Лемма 3 (ср. [2, гл. VII, предложение 4.8]). Пусть $\left(R_{n}\right)_{n=0,1, \ldots}$ - дискретный прочесс Бесселя. Выберем произвольное челое а $>0$ и рассмотрим момент $l_{a+}(R)$. Прочесс $\left(R_{n}\right)$, остановленный в этот момент, обладает симметричностью распределения в следуюшем смисле:

$$
\left(R_{n}\right)_{n \leqslant l_{a+}(R)} \stackrel{\text { law }}{=}\left(a-R_{l_{a+}(R)-n}\right)_{n \leqslant l_{a+}(R)} .
$$

Д о к а 3 а т е л ь с т в о. Легко видеть, что у рассматриваемых процессов совпадают множества траекторий. Это все траектории $\left(\omega_{n}\right)_{0 \leqslant n \leqslant N} \in \Omega_{N}^{+}, N>0$, такие, что $\omega_{0}=0, \omega_{N}=a$ и для каждого $n \leqslant N$ выполнены неравенства $0 \leqslant \omega_{n} \leqslant a$. Докажем, что при фиксированном значении $l_{a+}(R)=N$ все эти траектории равновероятны. Это не следует сразу из определения 4, поскольку момент $l_{a+}(R)$ не является моментом остановки. Но равенство $l_{a+}(R)=N$ эквивалентно тому, что $l_{a+1}(R)=N+1$, а величина $l_{a+1}(R)$ уже является марковским моментом. Поэтому из определения 4 следует, что каждая из этих траекторий имеет вероятность $c_{N+1}(a+2)$, где $c_{N+1}=1 / 2^{N+1}$. На этом доказательство леммы завершается.

Лемма $3^{\prime}$. Утверждение леммь 3 также справедливо для положительного прочесса Бесселя $\left(R_{n}^{+}\right)_{n=0,1, \ldots}$, остановленного в момент $l_{a}\left(R_{n}^{+}\right)$. 
Д о к а з а т е л ь с т в о. Воспользуемся определением 5 и применим лемму 3. Остается заметить, что при симметрии, рассматриваемой в лемме 3 , моменты $r_{0}$ и $l_{a}$ переходят друг в друга.

И еще одна вариация леммы 3.

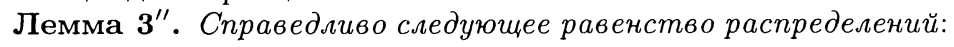

$$
\left(R_{n}\right)_{n \leqslant l_{a}(R)} \stackrel{\text { law }}{=}\left(a-R_{l_{a+}^{+}\left(R^{+}\right)-n}\right)_{n \leqslant l_{a+}\left(R^{+}\right)},
$$

где $\left(R_{n}\right)_{n=0,1, \ldots} u\left(R_{n}^{+}\right)_{n=0,1, \ldots}$ - это соответственно обычный и положительный процессы Бесселя.

Это утверждение также является прямым следствием определения 5 и леммы 3.

Лемма 4 (ср. [2, гл. VI, предложение 3.9]). Пусть $\left(R_{n}\right)_{n=0,1, \ldots}-$ - искретный прочесс Бесселя, а челое число а>0. Обозначим $J_{n}=\inf _{k \geqslant n} R_{k}$. Пусть некоторый случайный момент времени $\nu$ обладает свойством $R_{\nu}=J_{\nu} u \kappa$ тому же является моментом остановки относительно двумерного прочесса $\left(R_{n}, J_{n}\right)$. Тогда прочесс $\left(\widetilde{R}_{n}\right)_{n=0,1, \ldots}$, задаваемый формулой $\widetilde{R}_{n}:=R_{n+\nu}-R_{\nu}$, независим от $\left(R_{n}\right)_{n \leqslant \nu}$ и также является дискретным прочессом Бесселя. В частности, в качестве $\nu$ можно взять момент $r_{a-}(R)$.

Д о к а з а т е л ь с т в о. Представим процесс $\left(R_{n}\right)_{n=0,1, \ldots}$ в виде, указанном в определении 1 , т.е. пусть $R_{n}=2 M_{n}-S_{n}$, где $\left(S_{n}\right)_{n=0,1, \ldots}$ - некоторое простейшее симметричное случайное блуждание, а $\left(M_{n}\right)_{n=0,1, \ldots}$ - процесс его максимума. Как уже упоминалось, из возвратности случайного блуждания можно заключить, что $J_{n}=M_{n}$. Таким образом, мы получаем, что $S_{\nu}=M_{\nu}$. Из марковского свойства процесса $\left(S_{n}, M_{n}\right)_{n=0,1, \ldots}$ следует, что процесс $\widetilde{S}_{n}:=S_{n+\nu}-S_{\nu}$ также является простейшим симметричным случайным блужданием, причем независимым от $\left(S_{n}\right)_{n \leqslant \nu}$. Непосредственными вычислениями легко проверить соотношение $\widetilde{R}_{n}=2 \widetilde{M}_{n}-\widetilde{S}_{n}$, где $\widetilde{M}_{n}=\sup _{k \leqslant n} \widetilde{S}_{k}=M_{n-\nu}-M_{\nu}$. Это и доказывает лемму.

Лемма 5 (ср. [2, гл. VII, следствие 4.6]). Пусть $\left(R_{n}\right)_{n=0,1, \ldots}$ вновь обозначает дискретный прочесс Бесселя, а $\left(S_{n}\right)_{n=0,1, \ldots}$ - некоторое симметричное случайное блуждание. Зафиксируем произвольное челое $a>0$ и рассмотрим моменты $r_{a}(R)$ и $l_{a+}(S)$. Тогда следуюшие два прочесса равны по распределению:

$$
\left(S_{n}\right)_{n \leqslant l_{a+}(S)} \stackrel{\text { law }}{=}\left(a-R_{r_{a}(R)-n}\right)_{n \leqslant r_{a}(R)} .
$$

Д ок а з а т е л ь с т в о. Как и в лемме 3, сразу можно заметить, что у этих двух процессов совпадают носители. Их общий носитель состоит в точности из тех траекторий $\left(\omega_{n}\right)_{0 \leqslant n \leqslant N} \in \Omega_{N}, N>0$, для которых выполнены соотношения $\omega_{0}=0$, $\omega_{N}=a$ и $\omega_{n} \leqslant a$ для каждого $n$. Вновь аналогично доказательству леммы 3 , из того, что процессы $\left(R_{n}\right)$ и $\left(S_{n}\right)$ марковские, а также из определения 4 можно заключить, что при фиксированном значении $l_{a+}(S)=r_{a}(R)=N$ все эти траектории равновероятны.

Далее, как и при доказательстве леммы 4 , мы будем считать, что процесс Бесселя $\left(R_{n}\right)$ представлен в виде, указанном в определении 1 , т.е. $R_{n}=2 \widetilde{M}_{n}-\widetilde{S}_{n}$ для некоторого случайного блуждания $\left(\widetilde{S}_{n}\right)$ и его максимума $\left(\widetilde{M}_{n}\right)$. Нетрудно проверить, что в таком случае момент $r_{a}(R)$ имеет вид $r_{a}(R)=l_{a+}(\widetilde{S})$. Итак, мы убедились, что времена жизни рассматриваемых процессов также распределены одинаково, что и завершает доказательство.

Лемма 5'. Утверждение леммь 5 останется истинным, если рассматривать в ней положительный прочесс Бесселя и момент времени $l_{a}(S)$.

Д о к а з а т е л ь с т в о. Как и в лемме $3^{\prime}$, утверждение сразу следует из определения 5 и связи между величинами $r_{0}$ и $l_{a}$.

Теорема 2 (дискретная теорема Вильямса). Выберем произвольное челое число $b>0$. Пусть следуюшие четыре случайных элемента независимь: случайная величина а, имеющая дискретное равномерное распределение на отрезке $[0, b]$; симметричное случайное блуждание $\left(S_{n}\right)_{n=0,1, \ldots}$, выходящее из точки $b$; два дискретных прочесса Бесселя $\left(R_{n}^{1}\right)_{n=0,1, \ldots} u\left(R_{n}^{2}\right)_{n=0,1, \ldots}$.

Определим моменты времени $n_{1}$ u $n_{2}$ по формулам

$$
n_{1}=l_{b}\left(R^{1}\right):=\inf \left\{n \mid R_{n}^{1}=b\right\}, \quad n_{2}-n_{1}=l_{a}(S):=\inf \left\{n \mid S_{n}=a\right\} .
$$


Тогда прочесс $\left(R_{n}\right)_{n=0,1, \ldots}$, определенный по формуле

$$
R_{n}:= \begin{cases}R_{n}^{1}, & 0 \leqslant n \leqslant n_{1}, \\ S_{n-n_{1}}, & n_{1} \leqslant n \leqslant n_{2}, \\ a+R_{n-n_{2}}^{2}, & n_{2} \leqslant n,\end{cases}
$$

имеет распределение дискретного прочесса Бесселя.

Д о к а з а т е л в с т в о. Рассмотрим другой дискретный процесс Бесселя $\left(\widetilde{R}_{n}\right)_{n=0,1, \ldots}$ и представим его в виде, указанном в определении 1 , т.е. пусть $\widetilde{R}_{n}=2 \widetilde{M}_{n}-\widetilde{S}_{n}$ для некоторого случайного блуждания $\left(\widetilde{S}_{n}\right)_{n=0,1, \ldots}$ и его максимума $\left(\widetilde{M}_{n}\right)_{n=0,1, \ldots}$.

Рассмотрим момент $\widetilde{n}_{1}=l_{b}(\widetilde{R}):=\inf \left\{n \mid \widetilde{R}_{n}=b\right\}$ и обозначим значение $\widetilde{M}_{\widetilde{n}_{1}}$ через $\tilde{a}$. Как следует из леммы 2 , для каждого фиксированного вида траектории $\left(\widetilde{R}_{n}\right)_{n \leqslant \widetilde{n}_{1}}$ величина $\widetilde{a}$ распределена дискретно равномерно на отрезке $[0, b]$. Следовательно, ее безусловное распределение также равномерно и она не зависит от $\left(\widetilde{R}_{n}\right)_{n \leqslant \tilde{n}_{1}}$. K тому же из марковского свойства процесса $\left(\widetilde{R}_{n}\right)_{n=0,1, \ldots}$ следует, что процессы $\left(\widetilde{R}_{n}\right)_{n \leqslant \pi_{1}}$ и $\left(\widetilde{R}_{n}\right)_{n \geqslant \pi_{1}}$ независимы.

Далее, положим $\widetilde{n}_{2}=\inf \left\{n \mid n \geqslant \widetilde{n}_{1}, \widetilde{R}_{n}=\widetilde{a}\right\}$. Нетрудно видеть, что $\widetilde{M}_{n} \equiv \widetilde{a}$ для всех $n \in\left[\widetilde{n}_{1}, \widetilde{n}_{2}\right]$, а следовательно, процесс $\left(\widetilde{R}_{n}\right)_{\pi_{1} \leqslant n \leqslant \pi_{2}}$ имеет распределение симметричного случайного блуждания, выпушенного из точки $b$ в момент времени $\widetilde{n}_{1}$. Независимость этого случайного блуждания от величины $\widetilde{a}$ следует из марковости двумерного процесса $\left(\widetilde{S}_{n}, \widetilde{M}_{n}\right)$.

Момент $\widetilde{n}_{2}$ удовлетворяет условиям леммы 4, поэтому процесс $\left(\widetilde{R}_{n+\pi_{2}}-a\right)_{n=0,1, \ldots}$ является дискретным процессом Бесселя, независимым от $\left(\widetilde{R}_{n}\right)_{n \leqslant \pi_{2}}$. На этом доказательство теоремы заканчивается.

Теперь остановим построенный процесс $\left(R_{n}\right)_{n=0,1, \ldots}$ в момент $n_{3}=r_{b}(R):=$ $\sup \left\{n \mid R_{n}=b\right\}$ и применим к нему преобразование, состоящее в одновременной инверсии времени и применении отображения $x \mapsto b-x$ к фазовой переменной. На рис. 5,6 показано, как это происходит.

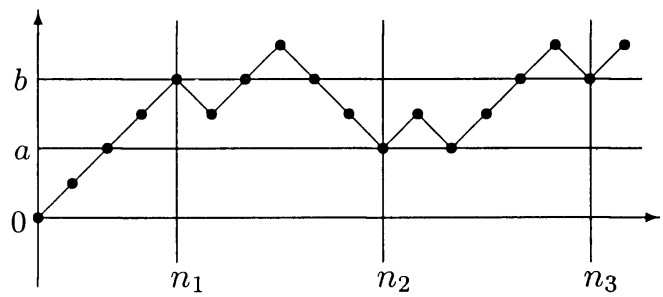

Рис. 5

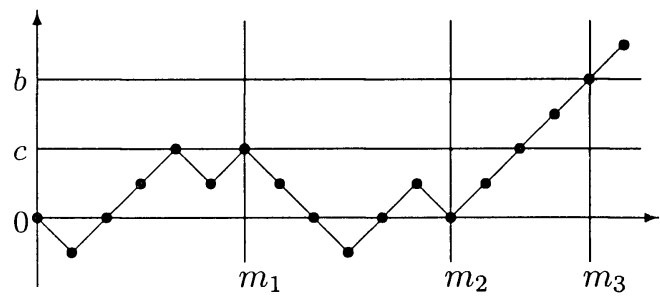

Рис. 6

На рис. 5 изображена одна из возможных траекторий процесса $\left(R_{n}\right)_{n=0,1, \ldots}$ и отмечены точки $n_{1}, n_{2}, n_{3}$. На рис. 6 показан процесс $\left(X_{n}:=b-R_{n_{3}-n}\right)_{n \leqslant n_{3}}$. Здесь 
$c=b-a, m_{1}=n_{3}-n_{2}, m_{2}=n_{3}-n_{1}, m_{3}=n_{3}$. С помощью доказанных выше лемм легко понять, что происходит с самим процессом $\left(R_{n}\right)_{n \leqslant n_{3}}$ и его частями при такой инверсии.

Так, из леммы 5 следует, что сам процесс $\left(X_{n}\right)_{n \leqslant m_{3}}$ является простейшим симметричным случайным блужданием, остановленным в момент $l_{b+}$, а его отрезок $\left(X_{n}\right)_{n \leqslant m_{1}}$ есть случайное блуждание до момента $l_{c+}$. Согласно лемме $5^{\prime}$, процесс $\left(c-X_{n+m_{1}}\right)_{n \leqslant m_{2}-m_{1}}$ является положительным процессом Бесселя, рассматриваемым до момента $r_{c}$. А процесс $\left(X_{n+m_{2}}\right)_{n \leqslant m_{3}-m_{2}}$ есть ноложительный процесс Бесселя, остановленный в момент $l_{b+}$, что следует из леммы $3^{\prime \prime}$. Тем самым доказана следующая теорема.

Теорема 3 (дискретное разложение Вильямса). Зафиксируем некоторое целое число $b>0$ и зададим следующие четыре независимых случайных элемента: случайная величинас с дискретным равномерным распределением на отрезке $[0, b]$; простейшее симметричное случайное блуждание $\left(S_{n}\right)_{n=0,1, \ldots} ;$ два положительных дискретных прочесса Бесселя $\left(R_{n}^{+, 1}\right)_{n=0,1, \ldots} u\left(R_{n}^{+, 2}\right)_{n=0,1, \ldots}$.

Определим моменты времени $m_{1}, m_{2}$ и $m_{3}$ по формулам

$$
\begin{gathered}
m_{1}=l_{c+}(S):=\inf \left\{n \mid S_{n}=c+1\right\}-1, \quad m_{2}-m_{1}=r_{c}\left(R^{+, 1}\right):=\sup \left\{n \mid R_{n}^{+, 1}=c\right\} \\
m_{3}-m_{2}=r_{b+}\left(R^{+, 2}\right):=\inf \left\{n \mid R_{n}^{+, 2}=b+1\right\}-1 .
\end{gathered}
$$

Тогда прочесс $\left(X_{n}\right)_{n \leqslant m_{3}}$, определенньй по формуле

$$
X_{n}:= \begin{cases}S_{n}, & 0 \leqslant n \leqslant m_{1}, \\ c-R_{n-m_{1}}^{+, 1}, & m_{1} \leqslant n \leqslant m_{2}, \\ R_{n-m_{2}}^{+, 2}, & m_{2} \leqslant n \leqslant m_{3},\end{cases}
$$

имеет распределение простейшего симметричного случайного блуждания, остановленного в момент $\tau_{b+}$.

Автор выражает признательность своему научному руководителю Альберту Николаевичу Ширяеву за помощь и поддержку, а также рецензенту за ценные замечания.

\section{СПИСОК ЛИТЕРАТУРЫ}

1. Pitman J.W. One-dimensional Brownian motion and the three-dimensional Bessel process. - Adv. Appl. Probab., 1975, v. 7, № 3, p. 511-526.

2. Revuz D., Yor M. Continuous Martingales and Brownian Motion. Berlin: SpringerVerlag, 1999, 602 p.

3. Williams D. Decomposing the Brownian path. - Bull. Amer. Math. Soc., 1970, v. 76, p. $871-873$.

Поступила в редакцию

17.VIII. 2005

(C) $2005 \mathrm{r}$.

JAJTE R.*

\section{POINTWISE ERGODIC THEOREM FOR UNBOUNDED OPERATORS IN $\mathbb{L}_{2}$}

Приводится достаточное условие справедливости усиленного закона больших чисел для траекторий нормального неограниченного оператора. Это условие формулируется в терминах спектральной меры. Чтобы рассмотреть случай неограниченных операторов, мы переходим от инструментов классической арифметики (Чезаро) к методам суммирования Бореля.

* Faculty of Mathematics, University of Łódź, Banacha 22, PL-90-238 Łódź, Poland; e-mail: rjajte@math.uni.lodz.pl 